\title{
Vận dụng phương pháp DEMATEL đánh giá rủi ro COVID-19 tới chuỗi cung ứng thực phẩm tại Việt Nam
}

\section{Applying DEMATEL method to assess COVID-19 risks to food supply chain in Vietnam}

\author{
Tôn Nguyễn Trọng Hiền ${ }^{1 *}$, Nguyễn Quỳnh Mai ${ }^{1}$

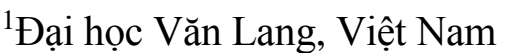 \\ "Tác giả liên hệ, Email: hien.tnt@vlu.edu.vn
}

\section{THÔNG TIN}

DOI: $10.46223 / \mathrm{HCMCOUJS.}$ econ.vi.17.1.1437.2022

Ngày nhận: 17/02/2021

Ngày nhận lại: 28/02/2021

Duyệt đăng: 09/03/2021

Tù khóa:

COVID-19; chuỗi cung ứng thực phẩm; DEMATEL; doanh nghiệp vận tải Việt Nam
Keywords:

COVID-19; food supply chain; DEMATEL; Vietnam logistics companies

\section{TÓM TẮT}




\section{Giới thiệu}

Cuộc khủng hoảng đại dịch Corona vi-rút 2019 (COVID-19) ngày càng sâu rộng khiến các quốc gia tạm thời đóng cửa giao thương giãn cách xã hội. Đại dịch đã có tác động đáng kể đến chuỗi cung ứng toàn cầu và tiếp tục ảnh hưởng đến các doanh nghiệp ngành thực phẩm hoạt động trên toàn thế giới. Việt Nam không ngoại lệ, trong khi Việt Nam đã và đang làm tốt việc ngăn chặn đại dịch, do tính liên kết toàn cầu, nền kinh tế của Việt Nam vẫn bị ảnh hưởng dẫn đến thiệt hại và bộc lộ những rủi ro khi cung ứng các sản phẩm. Hoạt động giao thương tại Việt Nam bị ảnh hưởng rất lớn bởi sự bùng phát của dịch COVID-19. Trước hết tổng kim ngạch xuất khẩu nông lâm thủy sản của Việt Nam giảm 4\% so với cùng kỳ năm 2019 (Tran, 2020). Nhu cầu tiêu dùng giảm do người dân thắt chặt chi tiêu, có sự thay đổi hành vi tiêu dùng. Thống kê cho thấy tiêu dùng thức uống giảm $14.1 \%$; sữa và sản phẩm từ sữa tăng $10.3 \%$; thực phẩm đóng gói tăng $26.2 \%$ (Son Nam, 2020). Bên cạnh đó, tác động của đại dịch COVID-19 đến thị trường vận tải biển là rất rõ ràng đại dịch COVID-19 sẽ khiến nhu cầu vận tải đường biển thế giới năm 2020 giảm khoảng 30\%, hoạt động vận tải hàng hóa bằng đường biển có lúc gần như tê liệt (Nguyen, 2020).

Nhiều nghiên cứu quốc tế kịp thời để tìm giải pháp giảm thiểu tác động tiêu cực của đại dịch. Quan sát kết quả nghiên cứu được báo cáo trên cơ sở dữ liệu Scopus nghiên cứu về ảnh hưởng dịch bệnh đối với chuỗi cung ứng thực phẩm, chúng tôi ghi nhận nhóm các hướng nghiên cứu tiêu biểu như: Đo lường tác động của COVID-19 đến giá, cổ phiếu và lợi nhuận trong chuỗi cung ứng thực phẩm (Coluccia, Agnusdei, Miglietta, \& Leo, 2021; Höhler \& Lansink, 2020); Đảm bảo an toàn thực phẩm trong tình hình dịch bệnh (Kumar, Mangla, Kumar, \& Song, 2021; Rahman, Sharun, Jose, \& Dhama, 2020; Rizou, Galanakis, Aldawoud, \& Galanakis, 2020); Chiến lược, chính sách ứng phó và khả năng phục hồi (Ali et al., 2021; Chenarides, Manfredo, \& Richards, 2020; Tasie, Reardon, \& Belton, 2020); Đề xuất nguyên nhân và/hoặc giải pháp hạn chế gián đoạn chuỗi cung ứng (Aday \& Aday, 2020; Mahajan \& Tomar, 2020; Montenegro \& Young, 2020; Singh, Kumar, Panchal, \& Tiwari, 2020).

Xem xét khoảng trống trong nghiên cứu, chúng tôi nhận thấy rằng phân tích mối quan hệ đan xen giữa các yếu tố rủi ro bị bỏ qua trong các phương pháp đánh giá rủi ro truyền thống. Mặc khác, bối cảnh nghiên cứu tại Việt Nam, việc nghiên cứu đánh giá rủi ro COVID-19 tới chuỗi cung ứng thực phẩm vẫn chưa được tiến hành. Do đó mục tiêu nghiên cứu là đánh giá rủi ro COVID-19 tới chuỗi cung ứng thực phẩm tại Việt Nam thông qua việc xác định mức độ quan trọng các rủi ro và mối liên hệ giữa chúng bằng phương pháp DEMATEL (Decision Making Trial and Evaluation Laboratory).

\section{Cơ sở lý thuyết và mô hình nghiên cứu}

Như đã đề cập, nhiều nghiên cứu trước đó được tiến hành đánh giá ảnh hưởng COVID-19 mang lại, trong đó có những nghiên cứu đánh giá tác động của COVID-19 đối với chuỗi cung ứng thực phẩm. Hầu hết các nghiên cứu đều đồng tình đại dịch COVID-19 đã mang lại những tác động chưa từng có đối với ngành công nghiệp thực phẩm và làm gián đoạn chuỗi cung ứng. Logistics (vận tải) là hoạt động chính của chuỗi cung ứng hàng hóa, hoạt động này bị tác động tiêu cực là tất yếu, do đó những giải pháp nhanh chóng được đề xuất. Singh và cộng sự (2020) đề xuất mô hình có thể giúp phát triển chuỗi cung ứng thực phẩm linh hoạt và đáp ứng để phù hợp với nhu cầu khác nhau cũng như hệ thống vận tải kết hợp giúp tiếp cận khu vực bị cách ly. Thực phẩm tươi sống là nhóm thực phẩm dễ bị hư hỏng nếu như quá trình vận chuyển bị gián đoạn quá lâu, mặt khác nghiên cứu cho thấy các sản phẩm tươi và dễ hư hỏng được sản xuất hoặc thu hoạch trong đợt COVID-19 đầu tiên đã bị ảnh hưởng về mặt giá cả (Coluccia et al., 2021), do đó nhiều giải pháp nhằm giảm thiểu rủi ro trong trong chuỗi thực phẩm tươi sống nói riêng, nâng cao an toàn vệ sinh thực phẩm trong tình hình dịch bệnh nói chung được nghiên cứu (Kumar et 
al., 2021; Rahman et al., 2020; Rizou et al., 2020). Chenarides và cộng sự (2020) đề xuất thuyết quyền chọn thực (real option theory) tăng cường tính linh hoạt chuỗi cung ứng nhằm giảm thiểu tổn thất, trong khi đó, nhóm nghiên cứu tại Ân độ (Mahajan \& Tomar, 2020) đề xuất nhóm giải pháp, tập trung giải pháp kho bãi và chính sách thu mua hàng hóa tại địa phương.

Xem xét bao quát hơn, Aday và Aday (2020) và Hobbs (2020) phân tích các tác động của cú sốc từ phía cầu đối với chuỗi cung ứng thực phẩm, bao gồm hành vi mua sắm hoảng loạn của người tiêu dùng tại đối với các mặt hàng thiết yếu, sự thay đổi đột ngột trong cách tiêu dùng từ lĩnh vực dịch vụ thực phẩm sang các bữa ăn được chế biến sẵn và tiêu thụ tại nhà (Hobbs, 2020), cùng với đó là thay đổi lựa chọn các thực phẩm tốt cho sức khỏe, an toàn (Aday \& Aday, 2020). Các gián đoạn tiềm năng từ phía cung ứng đối với chuỗi cung ứng thực phẩm cũng được đánh giá, bao gồm tình trạng thiếu lao động, gián đoạn mạng lưới giao thông và ùn ứ hàng hóa tại biên giới. Riêng bài báo của Hobbs (2020) xem xét liệu đại dịch COVID-19 có ảnh hưởng lâu dài hơn đến bản chất của chuỗi cung ứng thực phẩm hay không, bao gồm sự phát triển của lĩnh vực giao hàng tạp hóa trực tuyến và mức độ mà người tiêu dùng sẽ ưu tiên các chuỗi cung ứng thực phẩm địa phương. Khi tồn tại những bất cập tại một số địa phương trong tình hình dịch bệnh, những gợi ý chính sách được đề xuất (Tasie et al., 2020; Thilmany, Canales, Low, \& Boys, 2020). Ngoài ra, việc phân tích giá cổ phiếu và thông tin từ các báo cáo tài chính để xem xét tác động của đại dịch đối với sự biến động giá cổ phiếu và lợi nhuận của các công ty trong chuỗi cung ứng thực phẩm cũng được xem xét (Höhler \& Lansink, 2020).

Nhìn chung, COVID-19 tác động lên toàn cầu, nhưng các vấn đề và mức độ mỗi quốc gia chịu ảnh hưởng lại không giống nhau (Milani, 2020). Đại dịch COVID-19 vẫn còn tiếp diễn và mang đến những mang đến những vấn đề mà chúng ta gọi là rủi ro có thể ảnh hưởng tới chuỗi cung ứng (Hoek, 2020), tuy nhiên khi xem xét rủi ro toàn diện trong chuỗi cung ứng thực phẩm, những vấn đề gặp phải vẫn chưa rõ ràng (Aday \& Aday, 2020). Vẫn chưa có nghiên cứu về mối quan hệ đan xen giữa các yếu tố rủi ro đối với chuỗi cung ứng thực phẩm toàn cầu, và chưa có nghiên cứu như vậy cho bất kỳ quốc gia nào. Tầm quan trọng trong quản trị rủi ro là xác định các rủi ro và phân tích mối quan hệ (Babu, Bhardwaj, \& Agrawal, 2020; Govindan \& Chaudhuri, 2016), một khi nhận thức và đánh giá được rủi ro sẽ mang lại khả năng giảm cả khả năng rủi ro xảy ra và tác động tiềm tàng của nó. Do đó, đánh giá rủi ro COVID-19 tới chuỗi cung ứng thực phẩm tại Việt Nam được thực hiện.

Trong nghiên cứu này, đánh giá rủi ro được xác định bằng phương pháp DEMATEL. Phương pháp DEMATEL lần đầu tiên được giới thiệu vào năm 1973 bởi Gabus và Fontenla, để phân tích mối quan hệ và sức ảnh hưởng giữa các vấn đề phức tạp chẳng hạn như phân biệt chủng tộc, bảo hộ lao động, nạn đói, chủng tộc, etc., (Li, Hu, Zhang, Deng, \& Mahadevan, 2014) với mẫu khảo sát không quá lớn. Một cách tổng quát, phương pháp DEMATEL là một phương pháp luận có thể được sử dụng để nghiên cứu và giải quyết các nhóm vấn đề phức tạp và tác động qua lại. Sản phẩm cuối cùng của quy trình DEMATEL là một bản bản đồ quan hệ tác động các vấn đề cần giải quyết. Trong nhóm các phương pháp ra quyết định đa mục tiêu, DEMATEL tham khảo ý kiến chuyên gia tương tự như các phương pháp ISM, ANP, etc. Tuy nhiên, Wu (2008) cho rằng (ANP) đã được áp dụng thành công trong một số nghiên cứu, các nghiên cứu ANP đó không lý tưởng và chính xác vì không chỉ ra chính xác mối tương quan giữa các tiêu chí cần phân tích. Trong khi đó, phương pháp ISM một số điểm tương đồng với phương pháp DEMATEL, chẳng hạn như xác định mối quan hệ nguyên nhân và kết quả giữa một số yếu tố quyết định. Tuy nhiên, DEMATEL phân tích vấn đề chi tiết hơn, do đó Kumar và Dixit (2018) cho rằng ISM phân tích vĩ mô trong khi DEMATEL phân tích vi mô hơn. 


\section{Phương pháp nghiên cứu}

Phương pháp nghiên cứu được tiến hành qua các bước như sau:

(Bước 2.0) Thành lặp nhóm chuyên gia: Có 09 chuyên gia sẽ tiến hành đánh giá sơ bộ bộ tiêu chí. Họ đến từ 04 doanh nghiệp đang hoạt động tại Việt Nam, 02 doanh nghiệp vận tải, và 02 doanh nghiệp sản xuất. Để đảm bảo độ tin cậy, thành phần tham gia đánh giá đảm nhiệm vị trí phó phòng trở lên ở các bộ phận: Cung ứng, quản trị vận hành, đảm bảo rằng họ có cái nhìn bao quát.

(Bước 2.1) Chọn lọc rủi ro. Bao gồm tham khảo các nghiên cứu trước đây, chọn lọc, sau đó gửi chuyên gia đánh giá sơ bộ;

(Bước 2.2) Thiết lặp ma trận và tính toán trung bình cộng ma trận;

(Bước 2.3) Chuẩn hóa ma trận;

(Bước 2.4) Xây dựng ma trận quan hệ tổng quát;

(Bước 2.5) Xây dựng bản đồ quan hệ.

\subsection{Chọn lọc tiêu chí}

Chúng tôi quyết định chọn lọc rủi ro từ COVID-19 đối với chuỗi cung ứng thực phẩm thông qua các nghiên cứu trước đó để đảm bảo độ tin cậy và tránh thiên kiến trong quá trình chọn lọc dữ liệu. Chúng tôi sử dụng nguồn dữ liệu thứ cấp - cơ sở dữ liệu Web of Science (WoS) của Clarivate Analytics. Để sàn lọc lại một lần nữa những tạp chí đạt chất lượng tốt, chúng tôi so sánh kết quả tìm kiếm được với danh sách phân loại tạp chí (bản cập nhật mới nhất AJG 2018) biên soạn bởi Hiệp hội các trường kinh doanh Anh Quốc. Chi tiết tiêu chí tìm kiếm và kết quả được thể hiện bên dưới:

- Co sở dũ liệu: Web of Science;

- Ngày truy vấn: 10/01/2021;

- Tù khóa tìm kiếm: TITLE: (COVID * FOOD SUPPLY CHAIN);

- Kết quả tìm kiếm: 16 kết quả trả về (Bảng 1).

\section{Bảng 1}

Kết quả tìm kiếm tạp chí theo tiêu chí tìm kiếm

\begin{tabular}{c|l|l|c|}
\hline STT & \multicolumn{1}{c}{ DOI } & \multicolumn{1}{c}{ Tên tạp chí } & \multicolumn{1}{c}{$\begin{array}{c}\text { AJG } \\
\mathbf{2 0 1 8}\end{array}$} \\
\hline 1 & $10.1007 /$ s13197-020-04942-0 & $\begin{array}{l}\text { Journal of Food Science And Technology- } \\
\text { Mysore }\end{array}$ & $\mathbf{3}^{*}$ \\
\hline 2 & $10.1111 /$ ajae.12158 & $\begin{array}{l}\text { American Journal of Agricultural } \\
\text { Economics }\end{array}$ \\
\hline 3 & $10.1002 /$ agr.21678 & Agribusiness & \multirow{2}{*}{$\mathbf{2}^{*}$} \\
\hline 4 & $10.1093 /$ fqsafe/fyaa024 & Food Quality And Safety & $\begin{array}{l}\text { Applied Economic Perspectives and } \\
\text { Policy }\end{array}$ \\
\hline 5 & $10.1002 /$ aepp.13121 & $10.1080 / 1059924 X .2020 .1815623$ & Journal of Agromedicine \\
\hline 7 & $10.1002 /$ aepp.13139 & $\begin{array}{l}\text { Applied Economic Perspectives and } \\
\text { Policy }\end{array}$ \\
\hline
\end{tabular}




\begin{tabular}{|c|c|c|c|}
\hline STT & DOI & Tên tạp chí & $\begin{array}{l}\text { AJG } \\
2018\end{array}$ \\
\hline 8 & 10.5304/jafscd.2020.101.015 & $\begin{array}{l}\text { Journal of Agriculture Food Systems And } \\
\text { Community Development }\end{array}$ & \\
\hline 9 & 10.1016/j.tifs.2020.06.008 & Trends in Food Science \& Technology & \\
\hline 10 & $10.1080 / 00207543.2020 .1792000$ & $\begin{array}{l}\text { International Journal of Production } \\
\text { Research }\end{array}$ & $3^{*}$ \\
\hline 11 & $10.1038 / \mathrm{s} 43016-020-0097-7$ & Nature Food & \\
\hline 12 & 10.5304/jafscd.2020.094.031 & $\begin{array}{l}\text { Journal of Agriculture Food Systems And } \\
\text { Community Development }\end{array}$ & \\
\hline 13 & 10.1111/cjag.12237 & $\begin{array}{l}\text { Canadian Journal of Agricultural } \\
\text { Economics }\end{array}$ & $2 *$ \\
\hline 14 & No & International Sugar Journal & \\
\hline 15 & 10.1016/j.tifs.2020.03.041 & Trends in Food Science \& Technology & \\
\hline 16 & 10.1108/CAER-04-2020-0056 & $\begin{array}{l}\text { A snapshot of food supply chain in Wuhan } \\
\text { under the COVID-19 pandemic }\end{array}$ & \\
\hline
\end{tabular}

Nguồn: Web of Science

Từ 16 bài viết từ cơ sở dữ liệu WoS, có 05 bài viết xuất bản trong tạp chí được AJG 2018 xếp hạng $2 *$ trở lên, là tạp chí được đánh giá cao trong cùng lĩnh vực nghiên cứu. Tiến hành tổng hợp các bài viết nêu trên, đọc, phân tích và tổng hợp chúng tôi có được rủi ro mang đến chuỗi cung ứng thực phẩm như sau:

\section{Bảng 2}

Rủi ro COVID-19 ảnh hưởng đến chuỗi cung ứng thực phẩm

\begin{tabular}{|c|c|c|}
\hline Ký hiệu & Rủi ro & Giải nghĩa \\
\hline $\mathbf{A}$ & $\begin{array}{l}\text { Hàng hóa không thể lưu } \\
\text { thông } \\
\text { (Hobbs, 2020; Tasie et al., } \\
\text { 2020; Mahajan \& Tomar, } \\
\text { 2020; Thilmany et al., 2020) }\end{array}$ & $\begin{array}{l}\text { COVID-19 mang lại rủi ro về gián đoạn trong lưu } \\
\text { thông hàng hóa. Đại dịch COVID-19 mang lại mối } \\
\text { nguy hiểm sức khỏe nghiêm trọng và các quốc gia } \\
\text { trên thế giới đã phản ứng với việc đóng cửa một } \\
\text { phần nền kinh tế của họ để làm chậm tốc độ lây } \\
\text { nhiễm, điều này ảnh hưởng đến vận nguyên liệu và } \\
\text { hàng hóa }\end{array}$ \\
\hline B & $\begin{array}{l}\text { Thiếu lao động } \\
\text { (Hobbs, 2020; Tasie et al., } \\
\text { 2020; Singh et al., 2020) }\end{array}$ & $\begin{array}{l}\text { Mối nguy thiếu hụt lao động trong phân phối, vận } \\
\text { chuyển thực phẩm do bệnh tật, cách ly, giãn cách xã a } \\
\text { hội. Sự thiếu hụt trầm trọng những vùng có dịch do lo } \\
\text { ngại lây nhiễm. Ngoài ra, gián đoạn hoặc bất ồn trong } \\
\text { kinh doanh do dịch bệnh gây áp lực về tìm kiếm } \\
\text { nguồn lao động thay thế kịp thời }\end{array}$ \\
\hline C & $\begin{array}{l}\text { Giảm thu nhập nông dân } \\
\text { (Tasie et al., 2020; Mahajan \& } \\
\text { Tomar, 2020) }\end{array}$ & $\begin{array}{l}\text { Nông thủy sản không thể tiêu thụ do lệnh giới } \\
\text { nghiêm ảnh hưởng đến nguồn thu của nông dân. Ví } \\
\text { dụ khác, thiếu vác-xin do chuỗi cung ứng thuốc bị } \\
\text { nghẽn cũng ảnh hưởng đến thu nhập nông dân }\end{array}$ \\
\hline D & Thay đổi hành vi tiêu dùng & $\begin{array}{l}\text { Nguy cơ có sự thay đổi đột ngột trong mô hình tiêu } \\
\text { dùng từ lĩnh vực dịch vụ thực phẩm, ngoài ra tồn tại }\end{array}$ \\
\hline
\end{tabular}




\begin{tabular}{|c|c|c|}
\hline Ký hiệu & Rủi ro & Giải nghĩa \\
\hline & (Tasie et al., 2020) & $\begin{array}{l}\text { hỗn loại trong nhu cầu tiêu dùng thực phẩm hàng } \\
\text { ngày }\end{array}$ \\
\hline $\mathbf{E}$ & $\begin{array}{l}\text { Tăng chi phí vận chuyển } \\
\text { (Singh et al., 2020) }\end{array}$ & $\begin{array}{l}\text { Giao hàng không kịp thời làm tăng chí phí phát sinh } \\
\text { và chi phí thay thế phương tiện vận tải khác nếu có }\end{array}$ \\
\hline $\mathbf{F}$ & $\begin{array}{l}\text { Thay đổi chính sách } \\
\text { (Tasie et al., 2020; Thilmany } \\
\text { et al., 2020) }\end{array}$ & $\begin{array}{l}\text { Doanh nghiệp cần những chính sách ổn định lâu } \\
\text { dài, trong khi đó, dịch bệnh mang theo khả năng } \\
\text { thay đồi liên tục chính sách điều chỉnh trong tình } \\
\text { hình mới }\end{array}$ \\
\hline
\end{tabular}

Nguồn: Tác giả tổng hợp

Sáu tiêu chí rủi ro kèm diễn giải được gửi cho các chuyên gia thẩm định sơ xem liệu rằng các rủi ro này có phù hợp trong ngữ cảnh Việt Nam không. Kết quả thẩm định 06 tiêu chí này được thông qua.

\subsection{DEMATEL}

\subsubsection{Thiết lặp ma trận và tính trung bình cộng ma trận}

Nhóm chuyên gia được yêu cầu so sánh cặp các rủi ro với nhau theo thang đánh giá mức độ ảnh hưởng như sau: ' 0 ' =' không ảnh hưởng'; ' 1 ' = 'ảnh hưởng rất nhẹ', ' 2 ' = 'ảnh hưởng vừa phải', ' 3 ' = 'ảnh hưởng mạnh', '4' = 'ảnh hưởng rất mạnh'). Ví dụ: Rủi ro A không có ảnh hưởng lên rủi ro $\mathrm{B}$ là ' 0 '; rủi ro $\mathrm{B}$ có ảnh hưởng rất ít lên rủi ro $\mathrm{A}$ là ' 1 '.

Sau khi nhận được kết quả từ nhóm khảo sát chúng tôi xây dựng ma trận. Với mỗi đánh giá của người khảo sát, 01 ma trận vuông $n \times n$ được thiết lặp, trong đó $n$ là số biến quan sát. Số lượng ma trận bằng số người tham gia khảo sát.

$$
\begin{aligned}
& A^{1}=\left[\begin{array}{llllll}
0 & 0 & 2 & 1 & 3 & 0 \\
1 & 0 & 1 & 0 & 3 & 0 \\
1 & 0 & 0 & 2 & 1 & 3 \\
4 & 2 & 4 & 0 & 1 & 3 \\
2 & 1 & 2 & 0 & 0 & 2 \\
3 & 3 & 3 & 3 & 3 & 0
\end{array}\right] \quad A^{2}=\left[\begin{array}{llllll}
0 & 0 & 3 & 1 & 3 & 0 \\
2 & 0 & 1 & 0 & 3 & 1 \\
1 & 0 & 0 & 2 & 1 & 3 \\
4 & 2 & 4 & 0 & 1 & 3 \\
2 & 1 & 3 & 0 & 0 & 1 \\
4 & 3 & 2 & 2 & 3 & 0
\end{array}\right] \quad A^{3}=\left[\begin{array}{llllll}
0 & 1 & 2 & 2 & 3 & 0 \\
3 & 0 & 1 & 0 & 3 & 2 \\
2 & 1 & 0 & 2 & 1 & 3 \\
4 & 2 & 4 & 0 & 1 & 4 \\
4 & 1 & 2 & 1 & 0 & 2 \\
3 & 2 & 4 & 4 & 3 & 0
\end{array}\right] \\
& A^{4}=\left[\begin{array}{cccccc}
0 & 0 & 3 & 2 & 3 & 0 \\
1 & 0 & 1 & 1 & 3 & 0 \\
1 & 0 & 0 & 2 & 1 & 3 \\
2 & 2 & 4 & 0 & 1 & 2 \\
2 & 1 & 2 & 0 & 0 & 2 \\
3 & 2 & 2 & 4 & 3 & 0
\end{array}\right] \quad A^{5}=\left[\begin{array}{lllccc}
0 & 1 & 3 & 1 & 2 & 0 \\
2 & 0 & 2 & 0 & 2 & 1 \\
1 & 0 & 0 & 2 & 2 & 3 \\
3 & 2 & 3 & 0 & 2 & 4 \\
3 & 1 & 2 & 1 & 0 & 1 \\
3 & 3 & 3 & 2 & 3 & 0
\end{array}\right] \quad A^{6}=\left[\begin{array}{llllll}
0 & 1 & 1 & 1 & 3 & 0 \\
2 & 0 & 1 & 1 & 3 & 0 \\
1 & 0 & 0 & 2 & 1 & 3 \\
3 & 2 & 3 & 0 & 1 & 3 \\
2 & 2 & 2 & 2 & 0 & 2 \\
4 & 3 & 4 & 4 & 2 & 0
\end{array}\right] \\
& A^{7}=\left[\begin{array}{cccccc}
0 & 2 & 2 & 2 & 2 & 3 \\
1 & 0 & 1 & 1 & 3 & 1 \\
2 & 0 & 0 & 2 & 1 & 3 \\
3 & 2 & 4 & 0 & 1 & 3 \\
2 & 2 & 2 & 1 & 0 & 2 \\
2 & 2 & 3 & 1 & 4 & 0
\end{array}\right] \quad A^{8}=\left[\begin{array}{llllll}
0 & 1 & 3 & 1 & 3 & 3 \\
3 & 0 & 2 & 2 & 3 & 1 \\
1 & 3 & 0 & 2 & 1 & 2 \\
4 & 2 & 3 & 0 & 1 & 2 \\
2 & 1 & 2 & 1 & 0 & 1 \\
3 & 3 & 2 & 3 & 3 & 0
\end{array}\right] \quad A^{9}=\left[\begin{array}{llllll}
0 & 2 & 1 & 0 & 2 & 1 \\
1 & 0 & 1 & 0 & 3 & 1 \\
2 & 1 & 0 & 2 & 1 & 4 \\
4 & 2 & 4 & 0 & 1 & 3 \\
2 & 1 & 2 & 0 & 0 & 2 \\
2 & 3 & 3 & 4 & 2 & 0
\end{array}\right]
\end{aligned}
$$


Ta có kết quả ma trận trung bình như sau:

$$
A=\left[\begin{array}{cccccc}
0,0000 & 0,8889 & 2,2222 & 1,2222 & 2,6667 & 0,7778 \\
1,7778 & 0,0000 & 1,2222 & 0,5556 & 2,8889 & 0,7778 \\
1,3333 & 0,5556 & 0,0000 & 2,0000 & 1,1111 & 3,0000 \\
3,4444 & 2,0000 & 2,4444 & 0,0000 & 1,1111 & 3,0000 \\
2,3333 & 1,2222 & 2,1111 & 0,6667 & 0,0000 & 1,6667 \\
3,0000 & 2,6667 & 3,0000 & 2,6667 & 2,8889 & 0,0000
\end{array}\right]
$$

\subsubsection{Chuẩn hóa (matrix D)}

Tính toán ma trận quan hệ trực tiếp ban đầu $\mathrm{D}$ được chuẩn hóa bằng công thức: $\mathrm{D}=\mathrm{AxS}$, trong đó, Ta có:

$$
S=\frac{1}{\max _{1 \leqslant i \leqslant n} \sum_{j=1}^{n} a_{i j}}
$$

3.2.3. Xây dựng ma trận quan hệ tổng quát (Total relation matrix T)

$$
D=\left[\begin{array}{llllll}
0,0000 & 0,0625 & 0,1563 & 0,0859 & 0,1875 & 0,0547 \\
0,1250 & 0,0000 & 0,0859 & 0,0391 & 0,2031 & 0,0547 \\
0,0938 & 0,0391 & 0,0000 & 0,1406 & 0,0781 & 0,2109 \\
0,2422 & 0,1406 & 0,1719 & 0,0000 & 0,0781 & 0,2109 \\
0,1641 & 0,0859 & 0,1484 & 0,0469 & 0,0000 & 0,1172 \\
0,2109 & 0,1875 & 0,2109 & 0,1875 & 0,2031 & 0,0000
\end{array}\right]
$$

Tính toán ma trận ảnh hưởng tổng quát $(\mathrm{T})$ bằng công thức: $\mathrm{T}=\mathrm{D}(\mathrm{I}-\mathrm{D})-1, \mathrm{I}$ là ma trận đơn vị.

$$
T=\left[\begin{array}{cccccc}
0,2229 & 0,1984 & 0,3518 & 0,2247 & 0,3638 & 0,2420 \\
0,3115 & 0,1250 & 0,2732 & 0,1664 & 0,3648 & 0,2140 \\
0,3534 & 0,2153 & 0,2562 & 0,3045 & 0,3126 & 0,3969 \\
0,5500 & 0,3472 & 0,4840 & 0,2336 & 0,4009 & 0,4584 \\
0,3724 & 0,2254 & 0,3552 & 0,2029 & 0,21888 & 0,2932 \\
0,5697 & 0,4091 & 0,5533 & 0,4153 & 0,5338 & 0,3204
\end{array}\right]
$$

\subsubsection{Xây dựng bản đồ quan hệ}

Giả sử $r$ và $c$ là $n \times 1$ và $1 \times n$ vectơ đại diện cho tổng các hàng và tổng các cột của ma trận quan hệ $\mathrm{T}$. Ta gọi $r_{i}$ là tổng ảnh hưởng bao gồm ảnh hưởng trực tiếp và gián tiếp được gây ra bởi nhân tố thứ $i ; c_{j}$ là tổng ảnh hưởng, bao gồm cả trực tiếp và gián tiếp lên nhân tố thứ $j$ từ các nhân tố khác. Khi $\mathrm{i}=\mathrm{j}$, tổng $\left(r_{i}+c_{j}\right)$ nói lên mức độ quan trọng của nhân tố i. Trong khi đó, hiệu $\left(r_{i}-c_{j}\right)$ nói lên mối quan hệ nhân quả của các yếu tố. Trường hợp $\left(r_{i}-c_{j}\right)>0$, nhân tố i là nhân tố nguyên nhân (cause); Khi $\left(r_{i}-c_{j}\right)<0$, nhân tố i là nhân tố kết quả (effect). 


\section{Bảng 3}

Phân tích tầm quan trọng và xếp hạng của rủi ro

\begin{tabular}{|l|r|r|}
\hline \multicolumn{1}{|c|}{ Rủi ro } & r + c & r - c \\
\hline Hàng hóa không thể lưu thông (A) & 3.9837 & -0.7763 \\
\hline Thiếu lao động (B) & 2.9753 & -0.0654 \\
\hline Giảm thu nhập nông dân (C) & 4.1126 & -0.4349 \\
\hline Thay đổi hành vi tiêu dùng (D) & 4.0216 & 0.9266 \\
\hline Tăng chi phí vận chuyển (E) & 3.8625 & -0.5267 \\
\hline Thay đổi chính sách (F) & 4.7266 & 0.8767 \\
\hline
\end{tabular}

Nguồn: Kết quả phân tích dữ liệu của tác giả

Kết hợp giá trị ngưỡng tính được (Threshold alpha value) $=0.3289$ và Bảng 3 ta có sơ đồ quan hệ như sau:

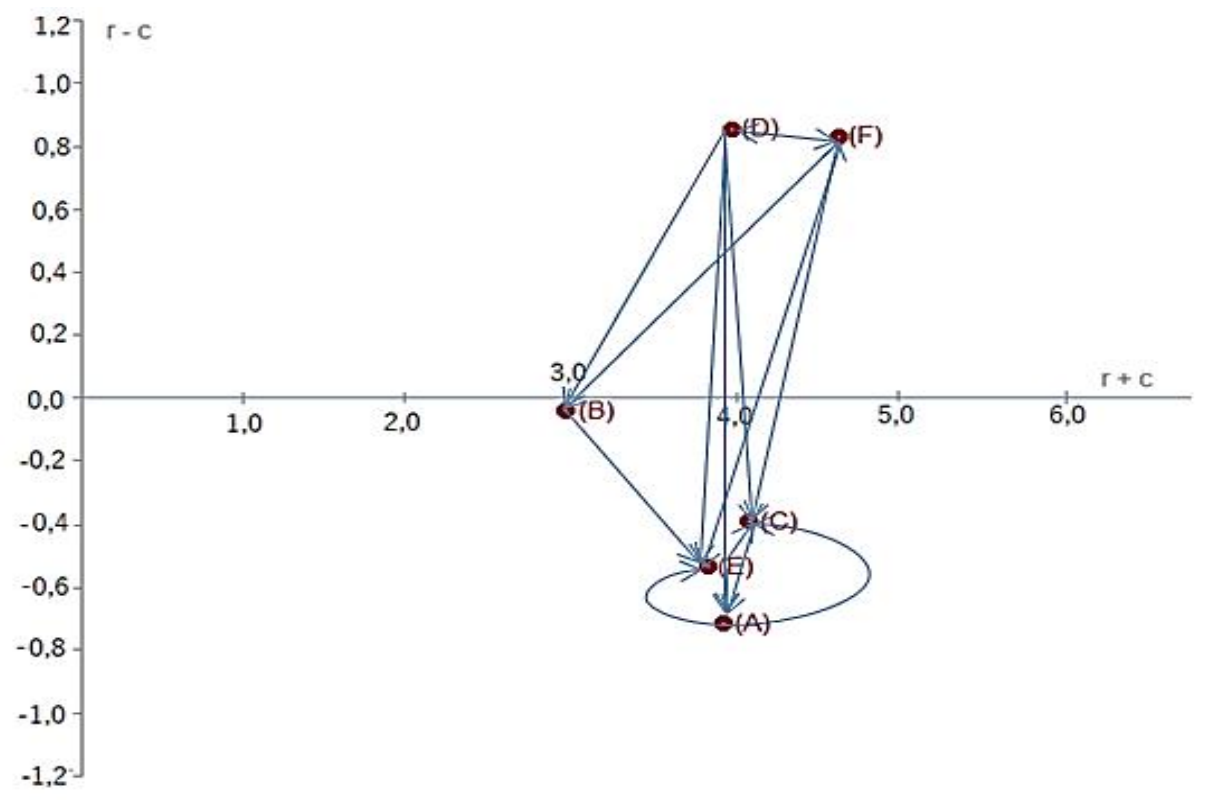

Hình 1. Bản đồ quan hệ các rủi ro COVID-19 tới chuỗi cung ứng thực phẩm Việt Nam Nguồn: Tác giả

\section{Kết quả nghiên cứu}

Kết quả phân tích từ Bảng 3 cho thấy, lo ngại về thay đổi chính sách $(\mathrm{F})$ được quan tâm nhiều nhất, kế đến rủi ro giảm thu nhập nông dân $(C)$, thay đổi hành vi tiêu dùng $(\mathrm{D})$, hàng hóa không lưu thông $(\mathrm{A})$, tăng chi phí vận chuyển $(\mathrm{E})$ là những rủi ro có mức độ quan trọng giảm dần. Trong khi đó vấn đề thiếu lao động vẫn chưa được quan tâm nhiều trong làn sóng dịch bệnh COVID. Cũng từ Bảng 3 , kết quả chỉ ra rằng rủi ro thay đổi hành vi tiêu dùng $(\mathrm{D})$ và khả năng có những thay đổi chính sách $(\mathrm{F})$ là nguyên nhân kéo theo những rủi ro bất cập khác, theo đó rủi ro $(\mathrm{F})$ là rủi ro có tác động mạnh nhất đến các rủi ro khác, dẫn đến hậu quả chịu tác động mạnh nhất là rủi ro $(\mathrm{A})$ - hàng hóa không thể lưu thông. 
Tuy thay đổi hành vi tiêu dùng $(\mathrm{D})$ và rủi ro thay đổi chính sách $(\mathrm{F})$ là rủi ro "chủ động", Hình 1 cho thấy, việc thay đổi chính sách từ nhà nước ảnh hưởng mạnh hơn, vẫn có khả năng ảnh hưởng đến người tiêu dùng thay đổi hành vi, trong khi đó, việc thay đổi chính sách hay không $(\mathrm{F})$ vẫn có khả năng chịu sự tác động từ việc nông dân bị giảm thu nhập $(\mathrm{C})$ và hành vi người tiêu dùng thay đổi $(\mathrm{D})$.

\section{Kết luận và gọii ý}

Nghiên cứu được thực hiện nhằm đánh giá rủi ro COVID-19 đối với chuỗi cung ứng thực phẩm tại Việt Nam. Kết quả cho thấy lo sợ về thay đổi trong chính sách và rủi ro thay đổi hành vi tiêu dùng có ảnh hưởng mạnh và có nguy cơ kéo theo các rủi ro khác. Hướng nghiên cứu tiếp theo cần thiết mở rộng mẫu nghiên cứu, phân tích mối liên hệ phức tạp của các yếu tố với nhau. Ngoài ra, có nhiều lý do khiến một số quốc gia có thể bị ảnh hưởng nặng nề hơn những quốc gia khác. Sự khác biệt trong phản ứng chính sách của chính phủ có thể là lý giải một số khác biệt, do đó cần thiết có những nghiên cứu phản ứng của chính sách trước dịch bệnh COVID-19 để có những điều chỉnh phù hợp, cũng như nghiên cứu ảnh hưởng thay đổi hành vi người tiêu dùng đối với dịch bệnh đến chính sách là cần thiết cho nghiên cứu tiếp theo.

\section{Tài liệu tham khảo}

Aday, S., \& Aday, M. (2020). Impact of COVID-19 on the food supply chain. Food Quality and Safety, 4(4), 167-180.

Ali, M., Suleiman, N., Khalid, N., Tan, K., Tseng, M., \& Kumar, M. (2021). Supply chain resilience reactive strategies for food SMEs in coping to COVID-19 crisis. Trends in Food Science \& Technology, 109(3), 94-102.

Babu, H., Bhardwaj, P., \& Agrawal, A. (2020). Modelling the supply chain risk variables using ISM: A case study on Indian manufacturing SMEs. Journal of Modelling in Management, 16(1), 215-239.

Coluccia, B., Agnusdei, G., Miglietta, P., \& Leo, F. D. (2021). Effects of COVID-19 on the Italian agri-food supply and value chains. Food Control, 123(5), Article 107839.

Chenarides, L., Manfredo, M., \& Richards, T. (2020). COVID-19 and Food supply chains. Applied Economic Perspectives and Policy, 43(1), 270-279.

Gabus, A., \& Fontela, E. (1973). Perceptions of the world problematique: Communication procedure, communicating with those bearing collective responsibility (DEMATEL Report No. 1). Geneva, Switzerland: Battelle Geneva Research Centre.

Govindan, K., \& Chaudhuri, A. (2016). Interrelationships of risks faced by third party logistics service providers: A DEMATEL based approach. Transportation Research Part E: Logistics and Transportation Review, 90(1), 177-195.

Hobbs, J. (2020). Food supply chains during the COVID-19 pandemic. Canadian Journal of Agricultural Economics/Revue Canadienne D'agroeconomie, 68(2), 171-176.

Hoek, R. (2020). Responding to COVID-19 supply chain risks - Insights from supply chain change management, total cost of ownership and supplier segmentation theory. Logistics, 4(4), 1-18.

Höhler, J., \& Lansink, A. (2020). Measuring the impact of COVID-19 on stock prices and profits in the food supply chain. Agribusiness, 37(1), 171-186. 
Kumar, A., \& Dixit, G. (2018). An analysis of barriers affecting the implementation of e-waste management practices in India: A novel ISM-DEMATEL approach. Sustainable Production and Consumption, 14(2), 36-52.

Kumar, A., Mangla, S., Kumar, P., \& Song, M. (2021). Mitigate risks in perishable food supply chains: Learning from COVID-19. Technological Forecasting and Social Change, 166(5), Article 120643.

Li, Y., Hu, Y., Zhang, X., Deng, Y., \& Mahadevan, S. (2014). An evidential DEMATEL method to identify critical success factors in emergency management. Applied Soft Computing, 22(11), 504-510.

Mahajan, K., \& Tomar, S. (2020). COVID-19 and supply chain disruption: Evidence from food markets in India. American Journal of Agricultural Economics, 103(1), 35-52.

Milani, F. (2020). COVID-19 outbreak, social response, and early economic effects: A global VAR analysis of cross-country interdependencies. Journal of Population Economics, 34(1), 223-252.

Montenegro, L., \& Young, M. (2020). Operational challenges in the food industry and supply chain during the COVID-19 pandemic: A literature review. In 7th International Conference on Frontiers of Industrial Engineering (ICFIE) (pp. 1-5). Singapore: IEEE Singapore SENS Chapter and Singapore Section.

Nguyen, T. (2020). Tác động của đại dịch COVID-19 đến thị truờng vận tải biển Việt Nam [The impact of the COVID-19 epidemic on the Vietnamese shipping market]. Retrieved January 15, 2021, from https://tapchitaichinh.vn/tai-chinh-kinh-doanh/tac-dong-cua-dai-dichcovid19-den-thi-truong-van-tai-bien-viet-nam-329698.html

Rahman, C., Sharun, K., Jose, B., \& Dhama, K. (2020). COVID-19 and food safety: Implications and opportunities to improve the food supply chain. Journal of Experimental Biology and Agricultural Sciences, 8(Spl-1-SARS-CoV-2), S34-S41.

Rizou, M., Galanakis, I., Aldawoud, T., \& Galanakis, C. (2020). Safety of foods, food supply chain and environment within the COVID-19 pandemic. Trends in Food Science \& Technology, 102(8), 293-299.

Singh, S., Kumar, R., Panchal, R., \& Tiwari, M. (2020). Impact of COVID-19 on logistics systems and disruptions in food supply chain. International Journal of Production Research, 59(7), 1-16.

Son Nam (2020). Dịch COVID-19 kéo ngành thức uống giảm, đẩy sản phẩm chăm sóc gia đình tăng [Pandemic COVID-19 has led to an reduction in beverages, and promoted home care products]. Retrieved January 15, 2021, from https://www.hcmcpv.org.vn/tin-tuc/dichCOVID-19-keo-nganh-thuc-uong-giam-day-san-pham-cham-soc-gia-dinh-tang-1491863982

Tasie, L., Reardon, T., \& Belton, B. (2020). "Essential non-essentials": COVID-19 policy missteps in Nigeria rooted in persistent myths about African food supply chains. Applied Economic Perspectives and Policy, 43(1), 205-224.

Thilmany, D., Canales, E., Low, S., \& Boys, K. (2020). Local food supply chain dynamics and resilience during COVID-19. Applied Economic Perspectives and Policy, 43(1), 86-104. 
Tran, D. (2020). Tác động của COVID-19: Đứt gãy chuỗi cung úng nông sản và giải pháp tái co cấu thị truòng [The impact of COVID-19: Breaking the supply chain of agricultural products and solutions to restructure the market]. Retrieved January 15, 2021, from https://vaas.vn/tieu-diem-binh-luan/tac-dong-cua-COVID-19-dut-gay-chuoi-cung-ung-nongsan-va-giai-phap-tai-co-cau.

$\mathrm{Wu}$, W. (2008). Choosing knowledge management strategies by using a combined ANP and DEMATEL approach. Expert Systems with Applications, 35(3), 828-835. 